\title{
Évaluation de la méthode impact-écho comme outil de détection de cavités
}

C. LEONARD

Laboratoire central des ponts et chaussées

Division MSRGI

58, bd Lefebvre

75732 Paris Cedex 15 christelle.leonard@lcpc.fr

\section{B. PIWAKOWSKI}

École centrale de Lille

Groupe électronique-

acoustique, BP 48

59651 Villeneuve-d'Ascq

Cedex

Bogdan.Piwakowski@ec-lille.fr

\section{SHAHROUR}

Laboratoire de mécanique de Lille

Polytech'Lille

Université des sciences et technologies de Lille 59655 Villeneuve-d'Ascq Isam.Shahrour@ polytech-lille.fr
Cet article présente un essai d'utilisation de la méthode impact-écho pour la détection de cavités souterraines. Cette méthode a largement a été appliquée avec succès pour la recherche de défauts dans des structures en béton. On montre dans cet article, par simulations numériques, que le transfert de la méthode impact-écho dans le domaine de la géophysique rencontre deux difficultés majeures. Premièrement, la forte absorption des sols par rapport au béton limite fortement le domaine d'application : la méthode est très peu performante dans des sols absorbants. Deuxièmement, une structure géologique est généralement stratifiée (à la différence du béton, qui est plus homogène) ce qui rend l'analyse des signaux impact-écho beaucoup plus délicate car la résolution est altérée. La méthode a toutefois été appliquée expérimentalement sur deux sites tests. Les résultats obtenus sont généralement en accord avec les valeurs attendues. Cependant, le phénomène observé semble plus provenir d'une perturbation des ondes de surface aux voisinage des cavités. Ce résultat, déjà noté par différents auteurs, est facilement mis en évidence par la méthode impact-écho.

Mots-clés: cavités, détection, méthode impact-écho, validations expérimentales, simulations numériques, fréquence de résonance.

\section{Use of impact-echo method for cavities location}

This paper presents a test of the application of the impact-echo method for the detection of underground cavities. This method is applied successfully for defects location in concrete

structures. By numerical simulations, it is shown in this paper. that its transfer in geophysics encounters two major difficulties. Firstly, the high absorption of the soil compared to the concrete strongly limits the applicability : the method gives a poor performance in absorbent soil. Secondly, a geological structure is generally layered (the concrete is more homogeneous) and the analysis of the impact-echo signals is much more difficult because the resolution is lower. However, the method was tested in two experimental sites. The results obtained are generally in agreement with the awaited values. But the phenomenon observed seerns coming from rather a disturbance of the surface waves the vicinity of cavities. This result, already noted by various authors, is easily underlined by the impactecho method.

Key words: cavities, detection, impact-echo method, experimental validations, numerical simulations, resonance frequency. 


\section{Introduction}

La détection de cavités souterraines par des méthodes géophysiques constitue, encore aujourd'hui, une tâche difficile. Malgré le large développement dé ces méthodes, chaque cas requiert une étude particulière et le recours à une méthode précise ou la combinaison de différentes méthodes (Fauchard et Pothérat, 2004: Beres et al., 2001). Des résultats positifs en terme de localisation de cavités ont pu être obtenus par sismique réflexion haute résolution (Branham et Steeples, 1988; Piwakowski et al,, 2002), par radar géologique (Beres et al., 2001; Chamberlain et al., 2000), par microgravimétrie (Crawford, 2000; Rybakov et al., 2001; Branston et al., 2003) ou par des mesures électriques (van Schoor, 2002). Cependant, ces méthodes ne sont pas toujours faciles à mettre en cuvre et requièrent un traitement des données relativement complexe.

Au travers d'études numériques et expérimentales, cet article présente une utilisation de la méthode impact-écho pour la détection de cavités souterraines. L'impact-écho est une méthode de contrôle non destructive qui a été initialement développée aux EtatsUnis, au Bureau national des normes et à l'université de Cornell, dans le milieu des années 80 pour la recherche de défauts dans le béton (Carino et al., 1986; Sansalone et Street, 1997; Sansalone et Carino, 1988). Des applications plus récentes de cette méthode se sont focalisées sur la détection de vides dans des gaines de précontrainte (Abraham et Cote, 2002; Forde et al., 2003), le contrôle non destructif de conduites en béton (Lin et Sansalone, 1994a, 1994b) et de structures de maçonnerie (Williams et al., 1997). Le principal avantage de cette méthode est sa simplicité de mise en œuvre, car elle ne nécessite qu'une seule source d'excitation et un seul récepteur, par point de mesure effectué.

Méthode simple et rapide, très efficace pour la recherche de vides dans des structures en béton, il est apparu intéressant d'étudier sa transposition dans le domaine de la géophysique pour détecter des cavités souterraines peu profondes (moins de $10 \mathrm{~m}$ ). Auparavant, il a semblé nécessaire d'analyser l'influence de l'absorption des sols sur les performances de la méthode, ainsi que les conséquences d'une application sur des structures géologiques stratifiées.

Cet article comprend trois parties: la première décrit la méthode; la deuxième présente une étude numérique concernant l'influence de l'amortissement du sol et de la stratification géologique sur les performances de la méthode; la dernière partie est consacrée à l'application expérimentale de la méthode sur des sites tests.

\section{2}

\section{Présentation}

\section{de la méthode impact-écho}

Suite à un impact mécanique $\mathrm{F}(\mathrm{t})$, appliqué au niveau de la surface d'une dalle en béton, une onde transitoire se propage dans la structure sous la forme d'ondes sphériques de compression et de cisaillement. Une onde de surface (ou onde de Rayleigh) existe aussi mais elle reste localisée à la surface de la dalle (son amplitude décroît exponentiellement en fonction de la profondeur) (Fig. 1a et annexe A). A l'intérieur de la dalle, le front d'onde se réfléchit à la fois sur les défauts (de dimensions suffisantes comparées à la longueur d'onde), et sur les bords de la structure, et réalise ainsi plusieurs allers-retours entre la surface, les bords de la dalle et les défauts. Un capteur localisé proche du point d'impact peut ainsi enregistrer les différents échos issus de l'impact mécanique. Une fois l'onde de Rayleigh dissipée, l'énergie des ondes de compression se propageant à l'intérieur de la dalle domine celle des ondes de cisaillement. Le signal y (t) enregistré par le capteur contient alors majoritairement les réflexions multiples (les échos) provenant des ondes de compression se propageant entre la surface de la structure et le défaut (Fig. 1b).

En l'absence de défaut, le signal y (t) enregistré par le capteur contient alors majoritairement les réflexions multiples provenant des ondes de compression se propageant entre la surface et le fond de la structure. Soit le temps $\tau_{c}$ nécessaire pour effectuer cet aller-retour, il peut être exprimé ainsi :

$$
\tau_{e}=2 \mathrm{e} / \mathrm{v}_{\mathrm{p}}
$$

où e indique l'épaisseur de la dalle en béton qui est considérée comme un milieu élastique, isotrope et homogène et où $v_{p}$ désigne la vitesse des ondes de compression.

Le signal y (t) enregistré alors contenant les arrivées périodiques des réflexions des ondes de compression espacées d'un intervalle de temps $\tau$ fournit un spectre périodique (Bracewell, 1965) de période spectrale $f_{\text {. }}$ désignée sous le nom de "fréquence de résonance » et écrite ainsi (Fig. 1c) :

$$
f_{e}=V_{p} / 2 e
$$

Sur les enregistrements impact-écho, il est généralement difficile de mesurer $\tau_{\mathrm{e}}$ dans le domaine temporel en raison de la superposition d'un nombre élevé d'arrivées d'ondes. Sous de telles conditions, les mesures de $\tau_{\varepsilon}$ sont effectuées dans le domaine fréquentiel, oủ les fréquences de résonance sont clairement identifiables. Ainsi, le passage du domaine temporel au domaine fréquentiel constitue le principe fondamental de la méthode.

En utilisant le même procédé, un vide localisé à une profondeur d sera caractérisé par une fréquence de résonance $f_{d}$ égale à :

$$
\mathrm{f}_{\mathrm{d}}=\mathrm{v}_{\mathrm{p}} / 2 \mathrm{~d}
$$

Les formules (1), (2) et (3) ne sont valides que pour des interfaces entre le béton et un matériau d'impédance acoustique $Z$ plus faible $\left(Z=\rho v_{\text {p }}\right)$ c'est-à-dire lorsque le coefficient de réflexion $\mathrm{R}$ est négatif ( $c$. annexe A). Dans le cas particulier où la réflexion se ferait sur un matériau de plus forte impédance acoustique, le coefficient de réflexion serait positif et la fréquence de résonance $f_{e}$ serait alors égale à :

$$
\mathrm{f}_{\mathrm{e}}=\mathrm{v}_{\mathrm{p}} / 4 \mathrm{e}
$$

La méthode impact-écho exige un calibrage afin de pouvoir calculer la profondeur du défaut. En effet, l'épaisseur de la dalle e étant connue, si la fréquence $f_{\text {. }}$ est mesurée, alors la vitesse $v_{p}$ peut alors être évaluée ắ partir de l'équation (1). En outre, si une fréquence $f_{d}$ est mesurée au-dessus d'un vide, la profondeur d du défaut peut être déterminée à partir de l'équation (3). Si aucun calibrage n'est possible (impossibilité de mesurer l'épaisseur de la dalle), une carte dans le domaine fréquentiel fournit des informations sur l'homogénéité de la structure et localise toutes les 


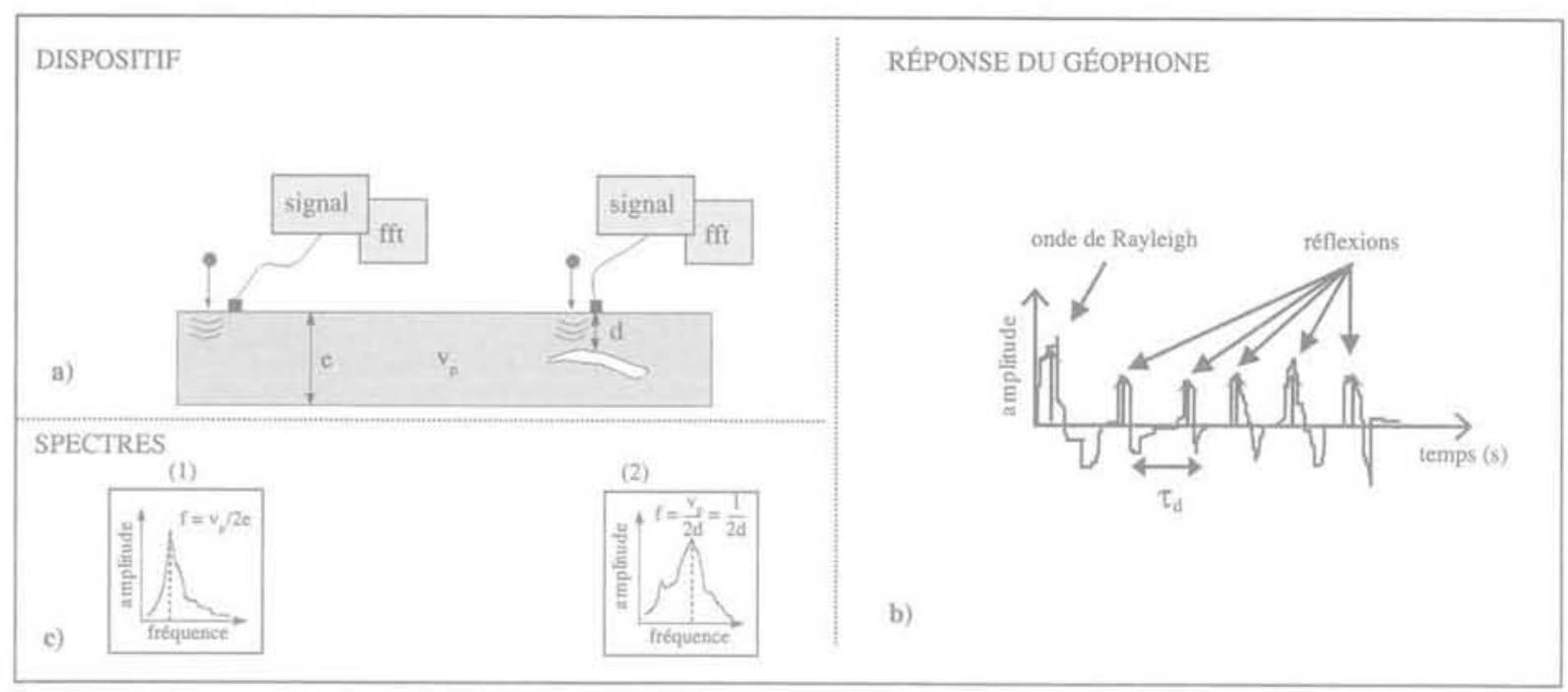

คG. 1 Principe de la méthode impact-écho.

a) Dispositif de mesure. b) Réponse temporelle d'un géophone localisé à la surface de la pièce à tester et proche de la source (au-dessus d'un défaut). c) Spectres de la réponse temporelle obtenus en l'absence (1) ou en présence d'un défaut (2) (extrait de Abraham et al., 2000).

zones anormales: un changement de fréquence du signal indiquerait alors la présence d'un défaut.

\section{3}

\section{Analyse des performances de la méthode impact-écho dans un contexte géologique}

L'utilisation de la méthode impact-écho pour la détection des cavités souterraines requiert auparavant une analyse de l'influence de la forte absorption des sols sur les performances de la méthode. En effet, l'absorption des ondes dans le béton peut être caractérisée par un facteur de qualité Q, traduisant l'amortissernent matériel (Aki et Richards, 1980; cf. annexe A), généralement compris entre 10 et 50 (Kharrat, 1997). Les sols sont beaucoup plus absorbants (Gutkowski et Dym, 1976), puisque le facteur de qualité $Q$ peut atteindre 3 , dans certaines argiles.

Dans ce qui suit est présentée une analyse par éléments finis des principaux facteurs pouvant influencer les performances de la méthode impact-écho, à savoir : l'absorption et la stratification des sols, la profondeur et la taille de cavité, le contenu fréquentiel de la force d'excitation F ( $\mathrm{t}$ ).

\section{1}

\section{Le modèle en éléments finis}

Les simulations numériques sont réalisées en utilisant le programme par éléments finis PECPLAS (Shahrour, 1992), qui combine une intégration de Newmark avec un amortissement de Rayleigh, permettant de ce fait d'introduire un coefficient de qualité Q.

Le modèle par éléments finis retenu correspond à une monocouche de craie, matériau que l'on observe sur le site (I) (voir \$ 4) dont les propriétés mécaniques ont pu être évaluées expérimentalement: $\mathrm{p}=2000 \mathrm{~kg} / \mathrm{m}^{3}, \mathrm{v}_{\mathrm{p}}=1666 \mathrm{~m} / \mathrm{s}, \mathrm{v}_{\mathrm{s}}=998 \mathrm{~m} / \mathrm{s}, \mathrm{Q}=10$.
Afin d'étudier le comportement tridimensionnel du système, les calculs sont effectués en symétrie de révolution (Moreaux, 1997). La cavité introduite correspond alors à un cylindrique de $6 \mathrm{~m}$ de hauteur et de rayon $\mathrm{R}$. La source ponctuelle, générant l'impact, est placée à la surface du modèle, sur l'axe de symétrie et au-dessus de la cavité, comme cela est indiqué sur la figure $2 a$.

Pour générer un impact, la source est modélisée par un vecteur force $F$ ayant une forme gaussienne (soit $\left.\exp \left[-\pi \sqrt{2} f_{p}(t-t s)^{2}\right]\right)$. Sa fréquence centrale $f_{p i}$ est choisie égale à $208 \mathrm{~Hz}$ et le temps d'inflexion ts est égal à $4,213 \mathrm{~ms}$. Notons que ces paramètres et la forme du vecteur force sont sélectionnés afin de reproduire au mieux la signature spectrale de l'impact généré par la source utilisée sur le terrain (Ricker, 1977).

Afin de reproduire correctement la propagation des ondes dans le modèle (éviter entre autres les réflexions sur les mailles, par exemple) (Moreaux, 1997), le domaine est constitué de mailles quadrilatères à 4 nœu ds de $1 \mathrm{~m}$ de côté et le modèle est pris suffisamment grand de façon à reproduire un domaine semiinfini. Le temps de calcul est fixé à $60 \mathrm{~ms}$, afin d'éviter les réflexions des ondes sur les bords du modèle (Fig. 2b). Enfin, pour assurer une bonne corrélation entre la discrétisation spatiale et temporelle, l'incrément de temps $\Delta t$ est choisi égal à $0,468 \mathrm{~ms}$.

\section{2}

\section{Analyse des résultats}

La réponse y $(x, t)$ en vitesse de 24 nœuds consécutifs du maillage situés à la surface du modèle entre $0 \mathrm{~m}$ et $23 \mathrm{~m}$ de la source ponctuelle, est présentée à la figure $2 \mathrm{~b}$. Nous observons principalement la propagation de l'onde de surface (SW) ainsi que les réflexions multiples des ondes de compression sur le toit de la cavité $\left(R_{n}\right)$. Ces ondes sont interprétées d'après leur vitesse de propagation. Les réflexions multiples $R_{p j}$, de très faibles amplitudes par rapport à l'onde directe SW, ne sont observables sur la figure $2 \mathrm{~b}$ que grâce à l'utilisation d'un contrôle automatique de gain. 


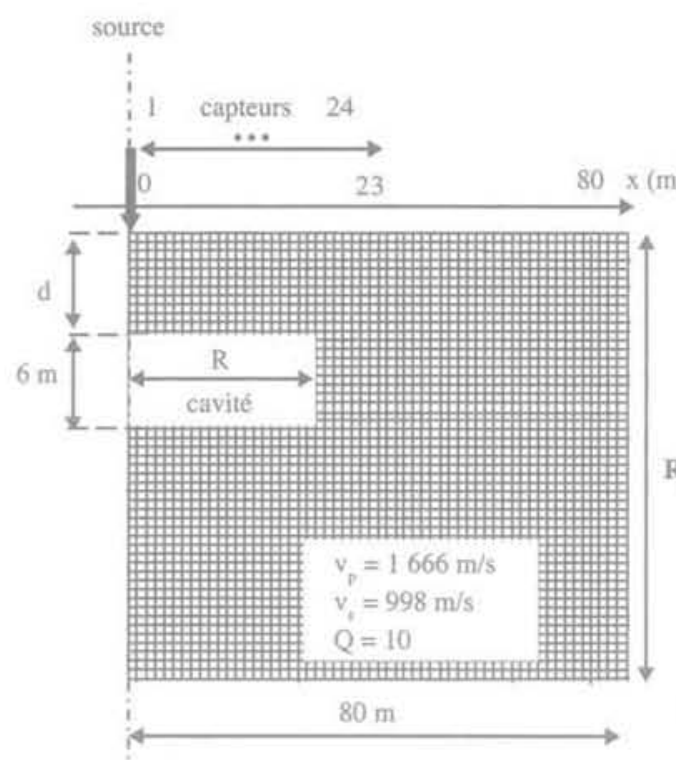

a)

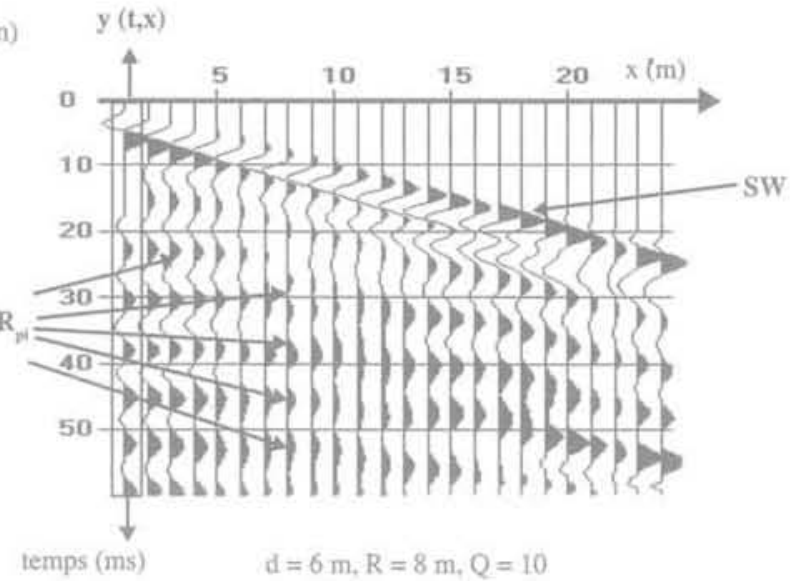

b)

AG.2 Modélisation par éléments finis d'une cavité cylindrique vide de rayon $\mathrm{R}$ localisée à une profondeur $d\left(R=8 \mathrm{~m}, \mathrm{~d}=6 \mathrm{~m}, \mathrm{Q}=10, \rho=2000 \mathrm{~kg} / \mathrm{m}^{3}, \mathrm{~V}_{\mathrm{f}}=1666 \mathrm{~m} / \mathrm{s}, \mathrm{V}_{\mathrm{s}}=998 \mathrm{~m} / \mathrm{s}\right)$, a) Maillage et position de la source et des géophones, b) Réponse en vitesse obtenue pour 24 géophones espacés de $1 \mathrm{~m}$, présentée avec un contrôle automatique de gain. SW et $R_{\mathrm{p}}$ représentent respectivement l'onde de surface et les réflexions multiples des ondes dé compression entre la surface du modèle et le toit de la cavité.

La réponse en vitesse de 24 nœuds consécutifs du maillage a été utilisée afin de mieux visualiser la propagation des ondes dans le modèle. Cependant, pour que les équations (1) à (4) soient valides, seul un capteur proche de la source peut être employé pour la méthode impact-écho. La figure 3a présente le signal y $(\mathrm{t})$, obtenu pour $\mathrm{x}=0$, correspondant à la première voie d'acquisition (trace 1 sur la figure $2 \mathrm{~b}$ ) sans contrôle automatique de gain: seule l'onde SW est observable. Sur la figure $3 \mathrm{~b}$, on observe que le spectre Y (f) du signal y (t) est dominé par l'onde de surface, son énergie est centrée autour de la fréquence centrale de la force d'excitation $\mathrm{f}_{\mathrm{Br}}$. La fréquence de résonance recherchée $\mathrm{f}_{\mathrm{d}}$ n'est pas visible $\left(f_{d}=\frac{2 v_{p}}{2 \mathrm{~d}}=139 \mathrm{~Hz}\right.$, équation 3$)$.

Pour l'observer, il est alors recommandé expérimentalement de saturer l'onde de surface et de n'étudier le signal y (t) qu'après son passage (Carino et al., 1986; Sansalone et Carino, 1988). Cette saturation permet d'enregistrer le signal utile de y $(\mathrm{t})$ avec une dynamique suffisante. Cependant, si une fenêtre temporelle $\mathrm{K}(\mathrm{t})$ est appliquée sur le signal y (t) (Fig. 3.c), le spectre $Y_{k}$ (f) du signal résultant $y_{\mathrm{K}}(\mathrm{t})$ montre un maximum qui est proche de la fréquence de résonance $f_{d f}$ recherchée (Fig. 3,d).

De façon à automatiser la procédure, une analyse temps-fréquence du signal y (t) est effectuée. Cette méthode provient du Laboratoire central des ponts et chaussées (Kahan, 1993; Abraham et al., 2000). Le principe consiste à étudier l'évolution du contenu fréquentiel du signal y $(\mathrm{t})$ au cours du temps en faisant glisser la

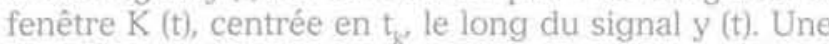
transformée de Fourier est calculée pour chaque signal fenêtré. L'analyse temps-fréquence consiste à juxtaposer les spectres obtenus $Y\left(f, t_{k}\right)$, l'échelle de temps indiquant alors le centre t des différentes fenêtres (Fig. 3e). Le spectrogramme est normalisé : l'amplitude maximale du spectre, représentée en blanc, vaut 1. La minimale, représentée en noir, vaut zéro. Sur la figure 3e, la fréquence de résonance $f_{f}$ des ondes de compression sur le toit de la cavité est clairement apparente dès la quatrième fenêtre (spectre obtenu à la figure $3 \mathrm{~d}$ ) alors que le début du signal est dominé par l'onde de surface. La valeur de $f_{d}$ obtenue $(139 \mathrm{~Hz})$ permet de retrouver la profondeur de la cavité $(\mathrm{d}=6 \mathrm{~m})$, en utilisant l'équation 3.

\section{3}

\section{Performances et limites de la méthode impact-écho}

La possibilité de détecter une cavité par la méthode impact-écho est évaluée à l'aice d'un critère de détection $\mathrm{C}_{\text {dec }}$ (cf. annexe $\mathrm{B}$ ). Ce dernier est défini à partir du nombre de fenêtres où la fréquence $\mathrm{f}_{\mathrm{d}}$ est observable dans l'analyse temps-fréquence ( 7 fenêtres sur 10 sur la figure 3 e) et de la qualité du spectre obtenu pour la quatrième fenêtre de l'analyse temps-fréquence (spectre de la figure $3 \mathrm{~d}$, pris comme référence car il possède un des meilleurs rapports signal/bruit). La qualité du spectre est jugée d'après la finesse du pic obtenu à la fréquence $f_{d}$ (bande passante $\Delta f$ à $-3 d B$ ) et le nombre d'harmoniques de $f_{d}$ observables (cf. annexe $B$ et Fig. 3d).

La figure 4 montre la variation du critère de détection $\mathrm{C}_{\mathrm{dcc}}$ en fonction de la profondeur d de la cavité et de sa taille $R$ et des paramètres adimensionnels $d / \lambda$ ( $\lambda$ étant la longueur d'onde) et AN, introduit par Piwakowski et al. (1998), qui définit le rapport de la surface de la cavité sur la surface de Fresnel (cf. annexe C). Les calculs sont réalisés pour deux coefficients de qualité, couramment rencontrés dans les sols: $Q=10$ et $Q=3$, ainsi qu'à titre indicatif, lorsque aucun amortissement n'est introduit $(\mathrm{Q}=\infty)$. 
a)

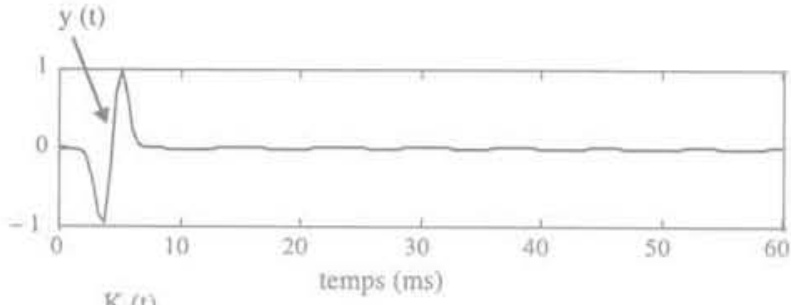

)

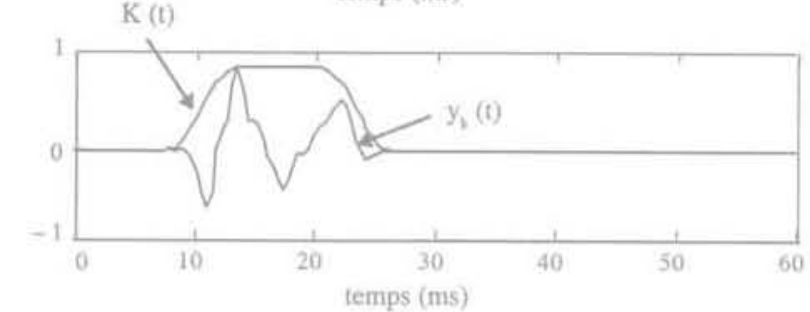

b)

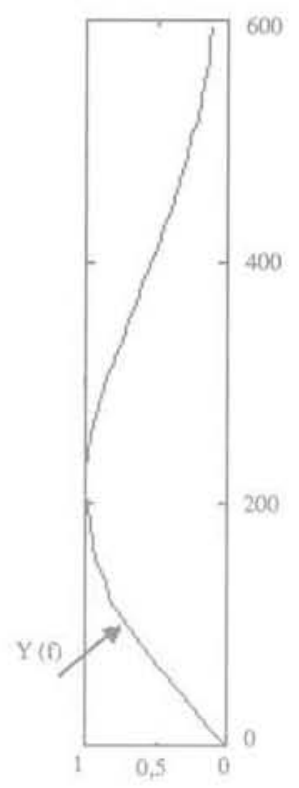

d)
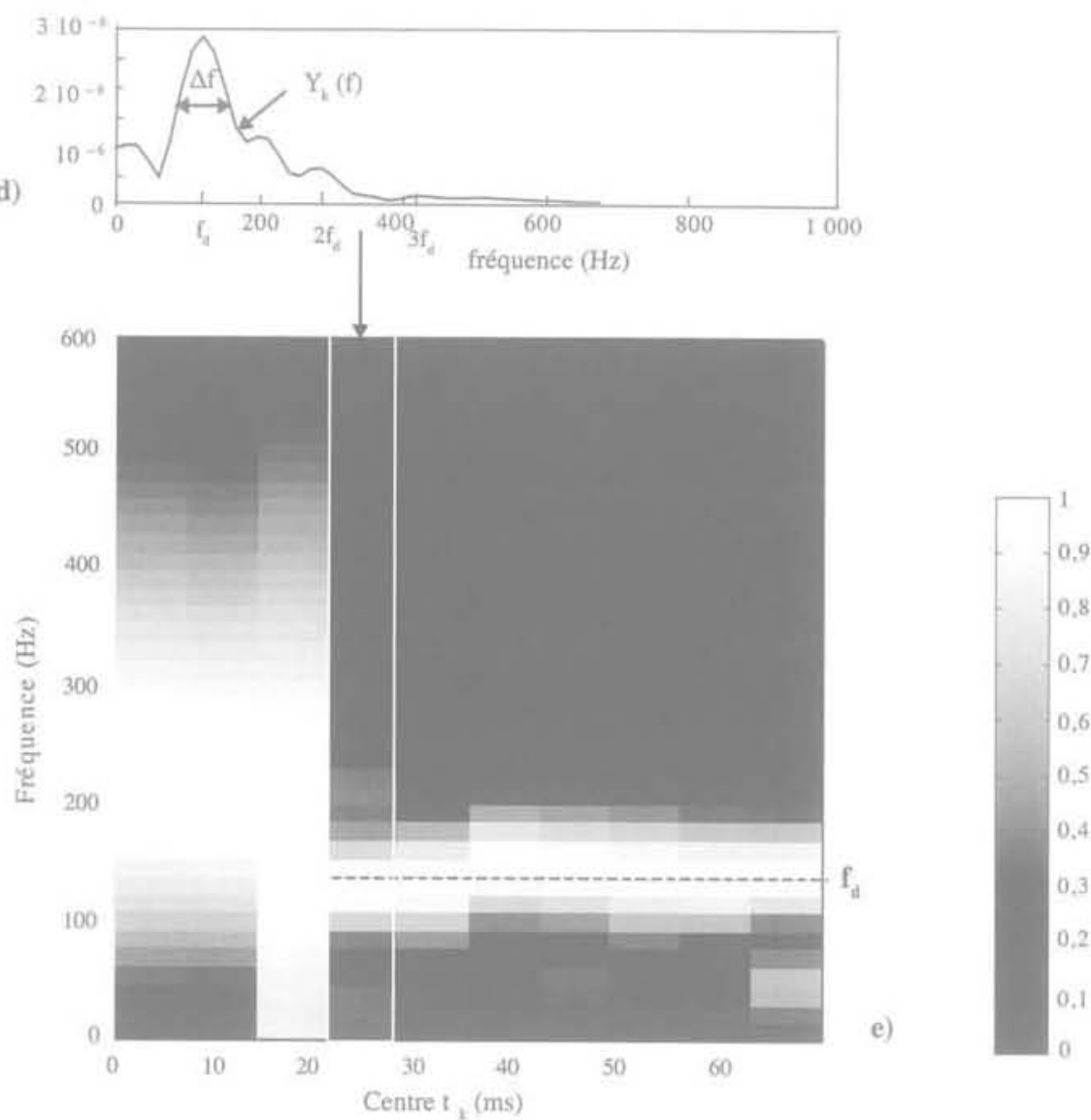

HG. 3 Résultats de la modélisation.

a) Trace $y$ [t, $x=0$ ) du signal présenté dans la figure $2 b$, sans contrôle automatique de gain. b) Spectra $Y$ (f) de $y$ (t). c) Signal $y_{1}(t)$ fenêtré par la fenêtre $K(t)$. d) Spectre $Y_{k}$ (f) de $y_{k}(t)$. e) Analyse temps-fréquence $Y\left(f, t_{k}\right)$, du signal présenté en al. La quatrième fenétre représente le spectre $Y_{t}$ (f) obtenu en d).

\section{3., 3. - 1 .}

\section{Influence de l'absorption du sol}

D'après l'évolution des courbes de la figure 4 , on constate sans surprise que le critère de détection $\mathrm{C}_{\text {die }}$ décroît en fonction l'absorption du sol (lorsque Q diminue), de plus, au voisinage de $d=0,75 \lambda(0,5 \lambda \leq d \leq 1 \lambda)$, le critère $\mathrm{C}_{\text {dec }}$ est maximal (Fig. 4a). En effet, lorsque la profondeur du toit de la cavité est proche de $\tilde{N} 2$ la force d'excitation $\mathrm{f}_{\mathrm{R}}$ coïncide avec la fréquence de résonance recherchée $f_{d}^{\prime}$ (cela peut être facilement démontré en posant $\lambda=v_{p} / f_{f i}$ et en utilisant l'équation 3) ce qui correspond aux conditions optimales d'utilisation de la méthode impact-écho.
De nombreuses études, présentées dans Léonard (2000), basées sur l'influence de la taille et de la profondeur de la cavité, de l'amortissement matériel du sol, du contenu fréquentiel de la source et du niveau de bruit, montrent que " $\mathrm{C}_{\text {dec }}=1$ » peut ètre considéré comme un bon seuil de détection, pour un coefficient de qualité Q supérieur ou égale à 3. Sous cette hypothèse et d'après la figure $4 \mathrm{~b}$, indépendamment de la valeur de $Q$, la détection d'une cavité devient quasiment impossible lorsque le coefficient d'anomalie AN, exprimant la taille de la cavité, devient inférieur à 0,15 $(\mathrm{R}=2 \mathrm{~m}$, pour $\mathrm{d}=6 \mathrm{~m})$. De plus, même pour une cavité de grande dimension $(\mathrm{R}=8 \mathrm{~m}, \mathrm{AN}=1)$, quelle que soit sa profondeur, sa détection est impossible lorsque le 

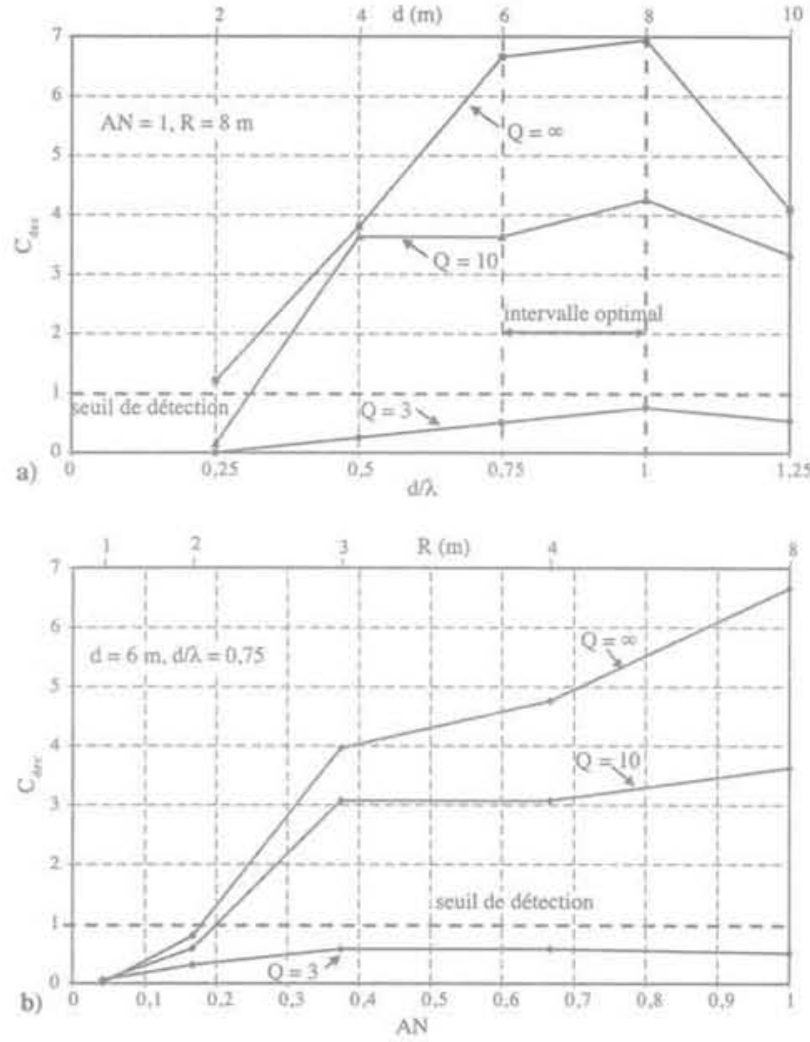

घ6.4 Évolution du critère de détection $\mathrm{C}_{\text {def }}$ en fonction de la profondeur de la cavité (a) et de la taille de cavité (b).

sol est très absorbant (caractérisé par un facteur de qualité du sol inférieur ou égal à 3, Fig. 4a). Sous cette condition, le critère $C_{\text {dec }}$ est en effet toujours inférieur à 1. Cependant, pour le cas particulier où le sol est caractérisé par un coefficient de qualitê Q égal à 10, on montre qu'une grande cavité $(\mathrm{AN}=1)$ devient détectable dès que sa profondeur dépasse $2,5 \mathrm{~m}$ (soit $\mathrm{d}=0,32 \lambda$, Fig. 4a). Si la cavité est moins étendue $(\mathrm{AN}<1)$ et localisée à une profondeur $\mathrm{d}=6 \mathrm{~m}$, elle devient détectable dès que sa taille atteint un rayon $R$ égal à $2 \mathrm{~m}$ (AN $>0,2$, Fig. 4b).

Pour conclure, cette étude montre que la méthode impact-écho est fortement dépendante de l'absorption des sols, de la taille et de la profondeur de la cavité (notamment du rapport taille/profondeur) et du contenu fréquentiel de la source utilisée. Cette remarque est bien sûr valable pour d'autres méthodes géophysiques (la sismique, par exemple), mais cela semble plus prononcé pour la méthode impact-écho.

\section{3 .2}

\section{Influence de la stratification}

L'influence de la stratification des sols est analysée au travers du site présenté dans le paragraphe 4.1 (Fig. $5 \mathrm{a}$ ). Le modèle géologique est caractérisé par une première couche d'argile $\left(p_{1}=1500 \mathrm{~kg} / \mathrm{m}^{3}\right.$, $\left.v_{p_{1}}=500 \mathrm{~m} / \mathrm{s}, v_{s_{1}}=263 \mathrm{~m} / \mathrm{s}, Q_{1}=5\right)$ suivie d'une couche de craie $\left(\rho_{2}=2000 \mathrm{~kg} / \mathrm{m}^{3}, v_{\mathrm{p} 2}=1666 \mathrm{~m} / \mathrm{s}, v_{\mathrm{S2}}=998 \mathrm{~m} / \mathrm{s}\right.$, $\mathrm{Q}_{2}=10$ ). La cavité (de $10 \mathrm{~m}$ de large et de $2 \mathrm{~m}$ d'épaisseur) est située à $8,50 \mathrm{~m}$ de profondeur, dans la craie. La nappe phréatique coïncide avec la fin de la craie, ce qui fournit un très bon contraste d'impédance acous- tique, favorable à la réflexion des ondes. Les fréquences de résonance correspondant aux différentes interfaces sont obtenues d'après les équations (2) et (4): - la fréquence de résonance $\mathrm{f}_{01}=\left(2 \mathrm{e}_{3} / \mathrm{v}_{\mathrm{p} 1}+2 \mathrm{~d} / \mathrm{v}_{\mathrm{p} 2}\right)^{-1}$ associée aux réflexions sur le toit de la cavité est égale à $63 \mathrm{~Hz}$ (éq. 2);

- la fréquence de résonance $\mathrm{f}_{02}=\left(2 \mathrm{e}_{3} / \mathrm{v}_{\mathrm{p} 1}+2 \mathrm{e}_{2} / \mathrm{v}_{\mathrm{p} 2}\right)^{-1}$ associée aux réflexions sur le fond de la craie blanche est égale à $46 \mathrm{~Hz}$ (éq. 2). En effet, le coefficient de réflexion sur la nappe phréatique est négatif;

- la fréquence de résonance $\mathrm{f}_{00}=(\mathrm{vp} / 4 \mathrm{~h})$ associée aux réflexions sur l'interface argile/craie est égale à $62,5 \mathrm{~Hz}$ (éq. 4).

Notons que, puisque les fréquences $\mathrm{f}_{01}$ et $\mathrm{f}_{03}$ coïncident, l'interprétation des résultats est plus délicate que dans le cas d'une monocouche.

D'après les recommandations de la section précédente, pour obtenir les conditions optimales d'utilisation de la méthode, la source doit couvrir un spectre compris entre 40 et $70 \mathrm{~Hz}$ (afin de coincider avec les fréquences de résonance recherchées). La longueur d'onde optimale $\lambda^{\prime}$ est donc obtenue pour vérifier l'équation $d=\lambda^{\prime} / 2$ (cf, section 3.3 .1 ), soit $\lambda^{\prime}=17 \mathrm{~m}$ $(\mathrm{d}=8,50 \mathrm{~m})$. D'après l'annexe $\mathrm{C}$, sous ces hypothèses, le coefficient d'anomalie AN est proche de 1. En considérant que le coefficient d'amortissement est supérieur à 5 , on peut conclure (Fig. 4.a) que la détection de la cavité est possible mais proche de ses limites (la courbe correspondant à $\mathrm{Q}=5$ est située entre celles obtenues pour $Q=3$ et $Q=10$ ).

Un profil impact-écho est simulé. Pour cela, soixante et un calculs sont réalisés en faisant avancer la source de $0,5 \mathrm{~m}$ à chaque fois (Fig. $5 \mathrm{~b}$ ). Cette dernière est introduite sous la forme d'une fonction de Gauss ayant une fréquence dominante $\mathrm{f}_{\mathrm{PJ}}=100 \mathrm{~Hz}$ (cf. \& 3.1 ) permettant de couvrir la bande $40 \mathrm{~Hz}-120 \mathrm{~Hz}$ et de cibler les différentes fréquences de résonance recherchées $\left(\mathrm{f}_{01}, \mathrm{f}_{02}\right.$ et $\left.f_{03}\right)$.

Une analyse temps-fréquence est alors effectuée. Afin de représenter le profil entier, pour chaque calcul, nous avons choisi de ne montrer que la $5^{e}$ fenêtre de l'analyse temps-fréquence (meilleur rapport signal/bruit) correspondant à l'intervalle d'observation [14 ms-46 ms]. La figure $5 \mathrm{c}$ présente alors l'évolution

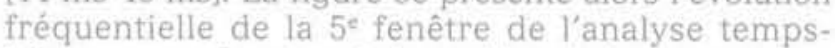
fréquence en fonction de la position de la source.

Le résultat obtenu montre une modification fréquentielle du signal au voisinage de la cavité. Dans la zone hors cavité, la fréquence observée semble être une moyenne des deux fréquences recherchées $\mathrm{f}_{02}$ et $\mathrm{f}_{03}$ (interférence des deux fréquences) alors qu'au-dessus du vide, on retrouve approximativement la fréquence de résonance $\mathrm{f}_{01}$ (associée aux réflexions sur le toit de la cavité).

A titre d'exemple, la figure $5 \mathrm{~d}$ présente le résultat obtenu pour une monocouche de craie. On remarque que la résolution spectrale est meilleure que dans le cas précédent. En effet, dans une structure stratifiée, les différentes réflexions et transmissions des ondes viennent atténuer le signal utile, ce qui n'est pas le cas dans une structure homogène où une seule réflexion existe ce qui permet d'obtenir un signal spectral centré autour de la fréquence de résonance recherchée.

Pour conclure, cette étude montre qu'une structure stratifiée induit la présence de plusieurs fréquences de résonance qui peuvent se masquer mutuellement ou 
a)

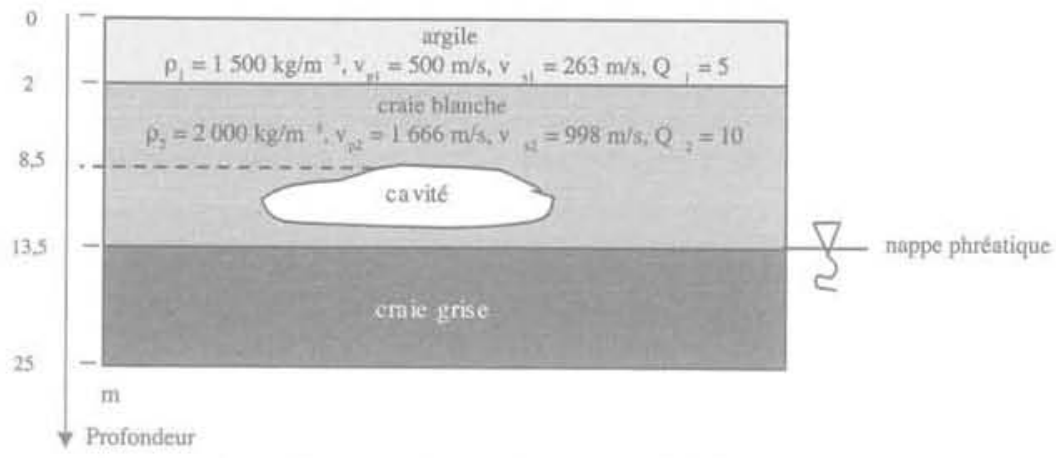

b)
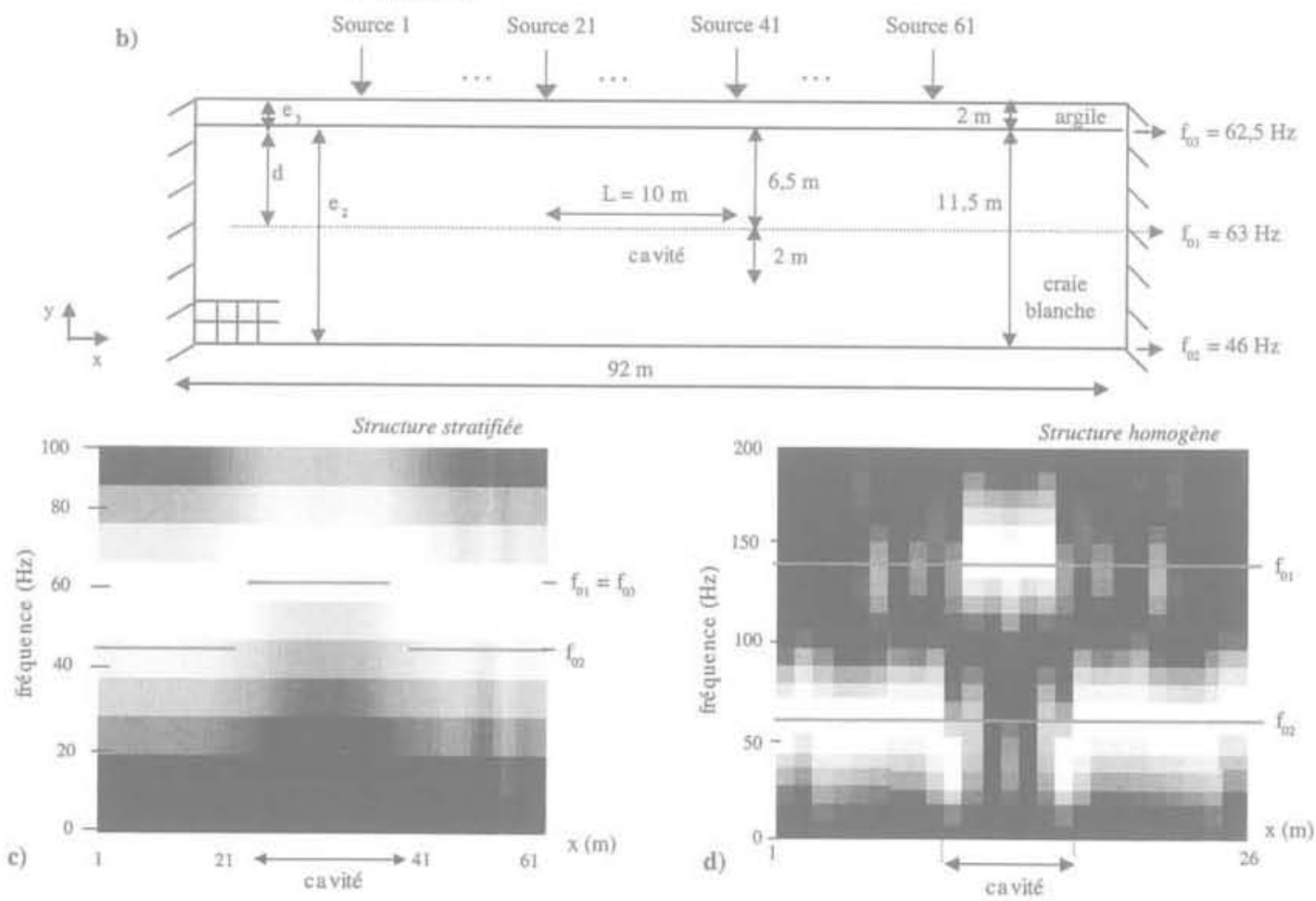

FiG.5 Simulation impact-écho sur une structure stratifiée.

a) Structure géologique du site. b) Modèle élément fini utilisé montrant les différentes positions des sources. c) Profi impact-écho obtenu : spectre $\mathrm{Y}(\mathrm{f})$ de la cinquième fenêtre de l'analyse temps-fréquence, présenté en fonction de la position de la source. d) Profil impact-écho obtenu pour une structure homogène (monocouche de craie, présentée à titre d'exemple).

interférer rendant l'interprétation des résultats plus délicate. D'autre part, la transmission des ondes au travers des clifférentes interfaces altère le signal et limite le nombre de réflexions multiples. La conséquence de ce type de propagation est similaire à une augmentation de l'absorption : les fréquences de résonance recherchées sont difficilement identifiables.

\section{4}

\section{Mise en œuvre expérimentale de la méthode impact-écho}

Après avoir étudié le potentiel de l'impact-écho au travers de modélisations numériques, nous allons mettre en œuvre cette méthode sur deux sites. La sismique réflexion haute résolution est utilisée sur le premier site comme outil d'aide à la décision pour les interprétations des résultats impact-écho. Elle permet de vérifier la bonne propagation des ondes sur le site (la présence d'argile peut atténuer le signal) et de valider la position des cavités.

\section{1}

\section{Site 1 : anciennes carrières abandonnées dans le Nord de la France}

Les mesures sont réalisées dans le Nord de la France sur un site présentant de nombreuses carrières abandonnées. Les cavités sont situées dans la craie à $8,50 \mathrm{~m}$ de profondeur. Les caractéristiques mécaniques et géologiques du site sont présentées sur la figure $5 \mathrm{a}$.

L'implantation du profil par rapport aux cavités est présentée sur la figure 6a. Les 48 premiers mètres (intervalle $\mathrm{AB}$ ) sont situés dans un endroit présentant de nombreux vides, tandis que le reste du profil (intervalle $\mathrm{BC}$ ) constitue notre référence car aucune cavité n'est recensée. Un puits a permis d'accéder aux cavités permettant de réaliser les mesures de vitesse de propagation ainsi que d'absorption (caractéristiques indiquées sur la figure 5a). La géométrie des cavités étant connue, le coefficient d'anomalie AN a pu être calculé le long du profil (Fig. 6d). Il approche la valeur de 1 dans l'intervalle (A-B) indiquant des cavités de dimen- 
sions suffisantes (correspondant aux hypothèses de la figure 4a). A noter que, d'après le paragraphe 3.3.2, la détection des cavités serait possible mais difficile.

\section{Astin:}

\section{Acquisition par la méthode impact-écho}

Un sismographe Geometrics Strata View possédant 48 voies a été utilisé pour l'acquisition cles données ainsi que des géophones ayant une fréquence de coupure de $40 \mathrm{~Hz}$. La source utilisée est une masse de $200 \mathrm{~kg}$ lấchée d'une hauteur de $2 \mathrm{~m}$ environ. L'avancement de la source comme l'espacement entre géophones est de $2 \mathrm{~m}$ soit une longueur du profil de $94 \mathrm{~m}$. Pour chaque enregistrement y (t), la source est placée à $1 \mathrm{~m}$ du géophone. Une analyse temps-fréquence est effectuée. Le spectre Y (f) sélectionné dans le profil impact-écho est celui obtenu dans la $4^{e}$ fenêtre de l'analyse temps-fréquence correspondant à l'intervalle [13 ms, $57 \mathrm{~ms}]$, car il possède le meilleur rapport signal/bruit. La figure $6 \mathrm{~b}$ présente les résultats obtenus. L'évolution du contenu fréquentiel est bien corrélé avec la présence des cavités. La fréquence observée dans l'intervalle (B-C) est égal à $45 \mathrm{~Hz}$, celle-ci coïncide avec la fréquence $\mathrm{f}_{02}$ associée aux réflexions sur la nappe phréatique située à $13,5 \mathrm{~m}$ de profondeur. Dans la zone des cavités (A-B), la fréquence fluctue entre 35 et $60 \mathrm{~Hz}$, la valeur la plus haute de l'intervalle étant égale à la fréquence de résonance des cavités $\mathrm{f}_{01}$ recherchée, la plus basse pourrait être associée à une interface plus profonde.

\section{a4k}

\section{Acquisition par la méthode sismique réflexion haute résolution}

L'acquísition a été réalisée avec un sismographe Geometrics Strata View et des géophones ayant une fréquence de coupure de $100 \mathrm{~Hz}$ qui permettent un filtrage basse fréquence des données sismiques. Une géométrie de type «tir en bout» a été utilisée ains: qu'un déport source - $1^{\text {er }}$ géophone (offset) de $17 \mathrm{~m}$ afin d'assurer une fenêtre d'observation optimale (optimum offset window) (Cook, 1965; Mari et al., 1997; Yilmaz, 1987) dans laquelle les réflexions provenant des "cibles " à détecter sont au mieux séparées des signaux constituant le bruit.

Un fusil de chasse "betsy-gun $»$, tirant des cartouches de type gros gibier de calibre 12 , semblable à celui présenté par Miller et al. (1986) a été utilisé comme source. Celle-ci est bien adaptée à des profondeurs d'investigation inférieures à 50 mètres et peut être actionnée directement depuis la surface, évitant ainsi la réalisation de forage.

Le profil sismique obtenu est présenté sur la figure $6 \mathrm{c}$. Dans la zone B-C sans cavité, l'absence de vide est indiqué par la présence d'un marqueur sismique, noté $\mathrm{C}$, non perturbé qui représente l'interface craie/marne situé à $25 \mathrm{~m}$ de profondeur (Fig. $5 \mathrm{a}$ ). Inversement dans la zone A-B présentant les cavités, le profil révèle une quasi-absence du marqueur $C$, ce dernier étant masqué par les vides. La détection des cavités est ici effectuée de manière indirecte, c'est-à-dire par la recherche de perturbations dans la coupe sismique induites par la présence des cavités (effet de masquage) (Piwakowski et al., 1998 et 2002).
Les résultats obtenus par cette méthode confirment la présence de vides dans l'intervalle A-B et une bonne propagation des ondes dans l'intervalle B-C, malgré la présence d'argile.

\section{Discussion}

Les résultats obtenus sur ce site par la méthode impact-écho sont moyennement satisfaisants. En effet, l'analyse théorique présentée au paragraphe 3.3.2 montre qu'en terrain stratifié la fréquence $\mathrm{f}_{0 z}$ associée aux réflexions sur la nappe phréatique située à $13,5 \mathrm{~m}$ de profondeur est difficilement observable, alors qu'aucun bruit ne vient perturber le signal. Il est donc fort probable qu'in situ, en contexte plus complexe, la fréquence détectée à $45 \mathrm{~Hz}$ ne provienne pas d'une interface aussi profonde. En analysant le contenu fréquentiel des signaux y $(t, x)$ dans l'intervalle $B-C$, on observe que cette valeur de $45 \mathrm{~Hz}$ coincide par ailleurs avec la fréquence dominante des ondes de surface (ground roll, cf. annexe A). Ces dernières, très énergétiques et dont la propagation est favorisée dans les structures multicouches, n'auraient donc pas été totalement supprimées lors de notre analyse temps-fréquence et masqueraient les réflexions multiples des ondes de compression. Les modifications spectrales observées sur la figure $6 \mathrm{~b}$ pourraient donc provenir d'une perturbation des ondes de surface au voisinage des cavités. Cette observation a été déjà notée par différents auteurs: Leparoux et al. (2000), Ormos et al. (1999). Abraham et Leparoux (2003) qui ont montré un effet de filtrage des ondes de surface par la cavité, à la fois dans le domaine temporel et fréquentiel. Par la mise en œuvre d'un profil impact-écho, on observerait alors facilement cette modification du contenu fréquentiel des ondes de surface aux voisinages des cavités. Ce phénomène, encore mal expliqué physiquement, fait actuellement l'objet de recherches au BRGM et au LCPC (division Reconnaissance et mécanique des sols).

\section{2}

\section{Site 2 : carrières de tuffeau dans le Maine-et-Loire}

Ce site est localisé en Maine-et-Loire, département qui possède de nombreuses carrières de tuffeau. La structure géologique du site, ainsi que la position du profil par rapport aux cavités sont présentées sur la figure 7. Les vides sont situés dans la couche de tuffeau, à des profondeurs comprises entre 2 et $3,5 \mathrm{~m}$. La position du profil est choisie de façon à couvrir une zone de référence sans cavité d'une longueur de $10 \mathrm{~m}$ (intervalle A-B). Le reste du profil surplombe les vides (intervalle B-C).

La possibilité d'accéder à la carrière a permis de réaliser une mesure des vitesses de propagation des ondes de compression entre la surface et le toit de la cavité ; la vitesse mesurée est de $500 \mathrm{~m} / \mathrm{s}$. Les fréquences de résonance attendues ont été calculées: - la fréquence de résonance $\mathrm{f}_{01}$ associée aux réflexions des ondes de compression sur le toit de la cavité est comprise entre 70 et $125 \mathrm{~Hz}$ (le toit de la cavité étant compris entre $2 \mathrm{~m}$ et $3,5 \mathrm{~m}$ );

- la fréquence de résonance $f_{\text {ce }}$ associée aux réflexions des ondes de compression sur l'interface tuffeau/sable située à $16,30 \mathrm{~m}$ de profondeur est estimée à $35 \mathrm{~Hz}$ (cette 


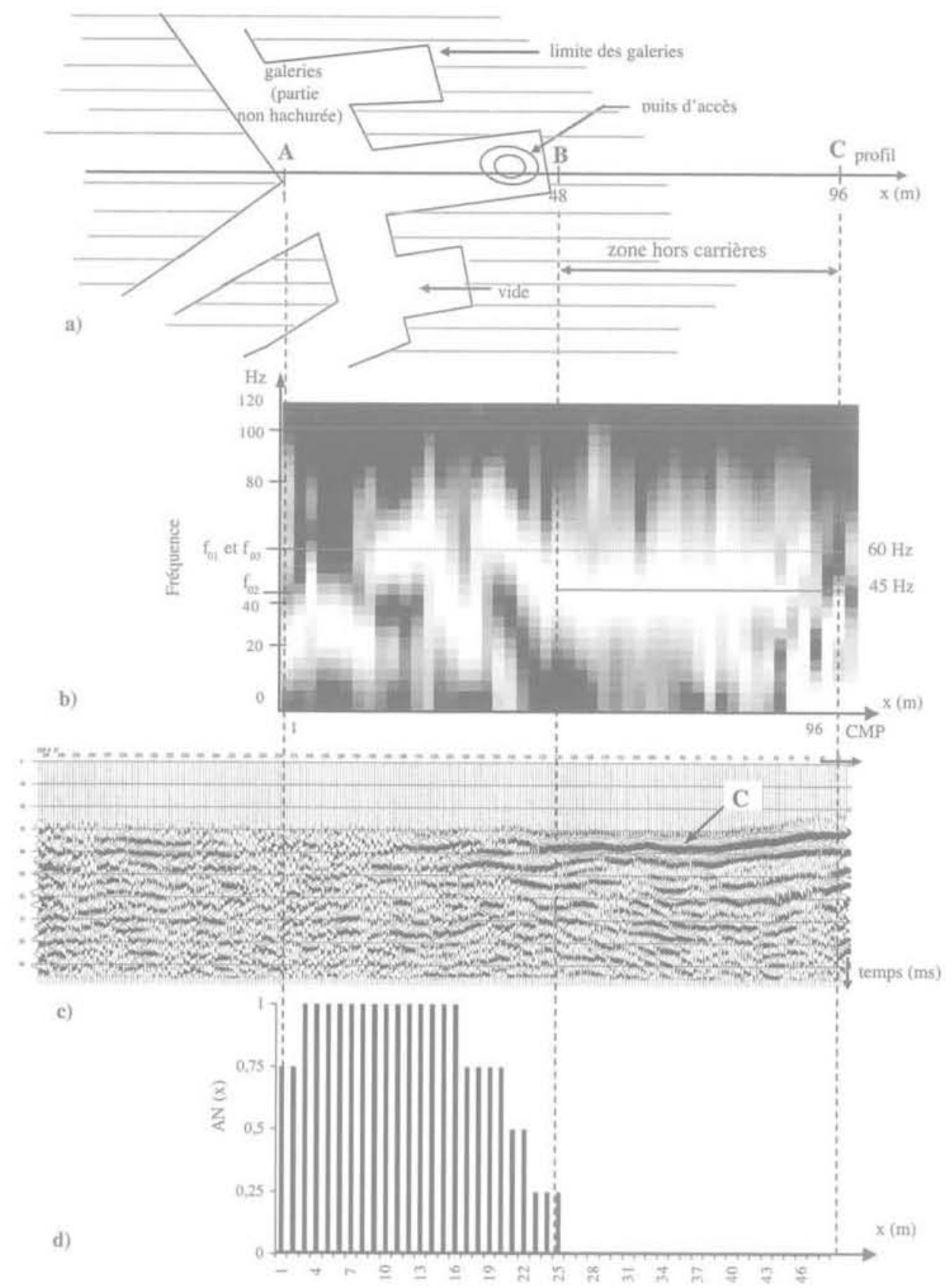

FiG.6 Validation expérimental de la méthode impact-écho sur le site test 1.

a) Localisation des galeries et du profil. Intervalle A-B : zone présentant les cavités; intervalle B-C: zone sans cavitẻ. b Spectre Y $(f, x)$ obtenu dans la $4^{\varepsilon}$ fenêtre de l'analyse temps-fréquence en fonction de la position de la source. c) Profi sismique réflexion haute résolution : le marqueur $\mathrm{C}$ représente l'interface craie grise/marne. d) AN (x).

interface ayant été détectée par sismique réflexion haute résolution, la vitesse des ondes de compression obtenue lors du traitement est égale à $1100 \mathrm{~m} / \mathrm{s}$ ).

Un sismographe Geometrics Strata View, possédant 48 voies et des géophones ayant une fréquence de coupure $100 \mathrm{~Hz}$ ont été utilisés pour l’acquisition des données. Une masse de $5 \mathrm{~kg}$ a été utilisée comme source, adaptée à des profondeurs relativement faibles (quelques mètres). L'espacement entre géophones et l'avancement de la source sont égaux à 0,5 m. La longueur du profil est de $28 \mathrm{~m}$.

La figure $7 c$ présente le spectre $Y(f, x)$ obtenu dans la $6^{e}$ fenêtre de l'analyse temps-fréquence (fenêtre optimale) en fonction de la position de la source correspondant à l'intervalle d'observation [ $25 \mathrm{~ms}$, $(56 \mathrm{~ms}]$. De même que le cas de la figure 6 , les modifications fré- quentielles observées sont bien corrélées avec la position des cavités. Une fréquence moyenne de $100 \mathrm{~Hz}$ est relevée dans l'intervalle B-C qui coïncide avec la fréquence de résonance $f_{01}$ attendue.

Cependant, dans l'intervalle A-B dépourvu de cavité, la fréquence obtenue de $70 \mathrm{~Hz}$ ne correspond pas à la fréquence attendue $f_{02}$ égale à $35 \mathrm{~Hz}$. Ceci est probablement lié à la fréquence de coupure des géophones utilisés qui supprime par filtrage le signal de $35 \mathrm{~Hz}$. Une analyse des ondes de surface montre par ailleurs que leur fréquence dominante est proche de $70 \mathrm{~Hz}$ (Fig. $7 \mathrm{~d}$ ) et coïncide alors avec la fréquence $\mathrm{f}_{01}$.

Les modifications spectrales observées sur la figure $7 \mathrm{c}$ pourraient donc provenir, comme pour le site 1 , d'une perturbation des ondes de surface au voisinage des cavités. 


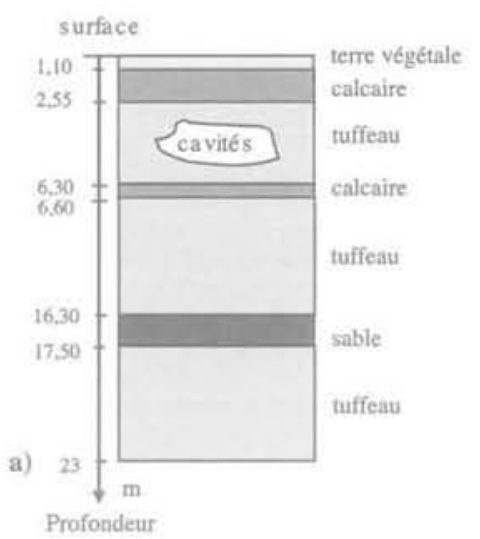

d)

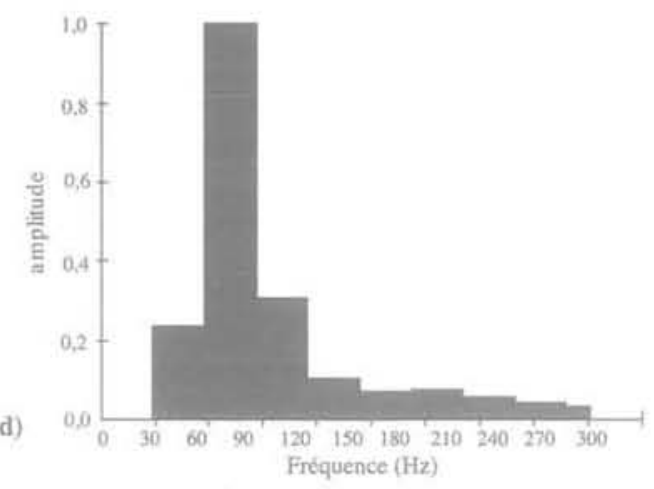

b)

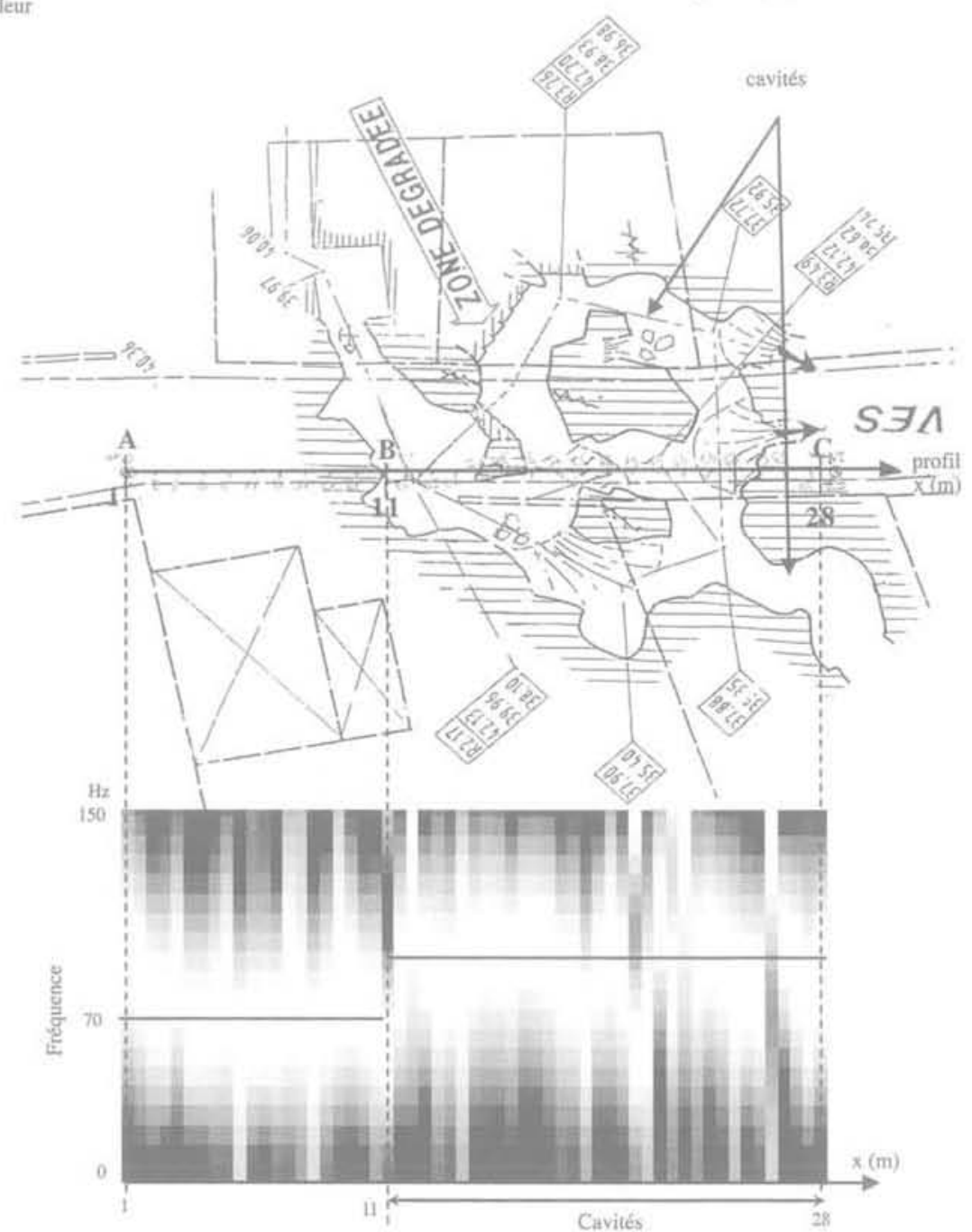

FiG.7 Validation expérimentale de la méthode impact-écho sur le site 2. a) Structure géologique du site. b) Localisation des cavités et du profil. Intervalle A-B: zone sans cavité. Intervalle B-C: zone avec cavités. c) Spectre Y $(f, x)$ obtenu dans la $6^{\circ}$ fenêtre de l'analyse temps-fréquence en fonction de la position de la source, d) Analyse du contenu fréquentiel des ondes de surface.

\section{Conclusion}

Cet article présente, aux travers de simulations numériques et de mesures expérimentales, l'étude de la transposition de la méthode impact-écho pour la détection de cavités souterraines.

Numériquement, on a montré dans cet article que le transfert de la méthode impact-écho vers la détection de cavités dans les sols rencontre deux difficultés majeures. Premièrement, la forte absorption des sols par rapport au béton limite fortement le domaine d'application: la méthode est très peu performante dans des sols absorbants $(Q<10)$. Deuxièmement, une structure géologique est généralement stratifiée (à la différence du béton, qui est plus homogène) ce qui rend l'analyse des signaux impact-écho beaucoup plus délicate, compte tenu des fréquences de résonance de la structure géologique qui altèrent la résolution de la méthode et gênent l'identification de la fréquence de résonance associée à la cavité. Dans un cas idéal pré- 
sentant une structure homogène peu absorbante $(Q>10)$ sans bruit, la méthode permet cependant de détecter des cavités dès que leur dimension dépassent $\mathrm{AN}=0,2$. On montre aussi que la méthode impact-écho est fortement dépendante du rapport taille/profondeur de la cavité et du contenu fréquentiel de la source utilisée (les conditions optimales d'utilisation de la méthode sont obtenues lorsque les fréquences de résonance impact-écho recherchées coïncident avec la fréquence d'excitation de la source).

Des mesures expérimentales ont été réalisées sur deux sites tests. Les résultats obtenus sont généralement en bon accord avec les valeurs attendues.

Toutefois nous avons observé, dans les deux cas étudiés, que le contenu fréquentiel des ondes de surface coïncide avec les fréquences impact-écho recherchées. Or, la propagation de ces ondes étant favorisée dans des structures stratifiées, leur niveau d'amplitude est beaucoup plus élevé que celui des réflexions des

\section{Annexes}

\section{A. Quelques notions de propagation d'onde}

La théorie de l'élasticité et le principe fondamental de la dynamique montrent que les ondes se propagent dans un milieu par deux mécanismes différents et donnent ainsi deux types d'ondes se propageant de manière indépendante: les ondes de compression ou longitudinales (onde P) et les ondes de cisaillement ou transversales (onde S) (Lavergne, 1986).

Dans un milieu solide, infini, homogène et isotrope, seules des ondes de volume se propagent : les ondes P.

avec pour vitesse $v p=\left(\frac{E(1-v)}{\rho(1+v)(1-2 v)}\right)^{1 / 2}$, pour lesquels le

déplacement des particules est parallèle au sens de propagation de l'onde et les ondes S

$\left(V_{s}=\left(\frac{E}{2 p(1+v)}\right)^{1 / 2}\right.$ ) pour lesquelles le déplacement est

perpendiculaire au sens de propagation de l'onde. Dans le cas d'un milieu semi-infini, limité par une surface libre (la surface du sol, par exemple), d'autres types de propagation existent au voisinage de la surface. Ce sont les ondes de surface qui pourront être soit des ondes de Love si le mouvement des particules est contenu dans un plan vertical perpendiculaire à la direction de propagation, soit des ondes de Rayleigh si le mouvement des particules est contenu dans un plan vertical parallèle à la direction de propagation. En présence d'une couche superficielle, des ondes analogues aux ondes de Rayleigh peuvent se propager à la surface. Ces ondes, appelées pseudo-Rayleigh, sont très énergétiques et génèrent un bruit important (ground roll) dans les enregistrements sismiques.

En présence d'interfaces planes et de grandes dimensions, les ondes sont partiellement transmises et réfléchies, Un coefficient de réflexion $\mathrm{R}$ peut être défini au niveau de cette interface pour les ondes de compression, à incidence normale, par la relation suivante :

$$
R=\left(Z_{2}-Z_{3}\right) /\left(Z_{1}+Z_{2}\right)
$$

ondes de compression. Il est donc fort probable que les fréquences recherchées soient masquées et que le phénomène observé provienne d'une perturbation des ondes de surface aux voisinage des cavités.

Finalement, indépendamment du type de phénomène détecté par la méthode impact-écho, l'approche proposée, rapide, facile à implanter et peu onéreuse peut fournir une aide dans la détection des cavités, si elle est associée une autre méthode de reconnaissance. La méthode impact-écho pourrait par exemple être utilisée pour "suivre» le long d'un profil la présence de cavités, détectées auparavant en un point donné par une autre technique, plus efficace mais plus onéreuse.

\section{PEMERCIDNENTS}

Les auteurs remercient la division RMS du LCPC de Nantes qui est à l'initiative de ce travail et qui a apporté un soutien scientifique et financier à cette étude.
$Z_{1}$ et $Z_{2}$ sont respectivement les impédances acoustiques du premier et du deuxième matériau traversés par l'onde de compression au niveau de cette interface $(\mathrm{Z}=\rho \mathrm{Vp})$.

En milieu homogène et élastique, le mouvement des particules s'atténue au cours de la propagation, du fait de l'expansion du front d'onde. L'amplitude des ondes diminue alors suivant une loi en $1 / \mathrm{r}$, r étant la distance du point d'observation au point source, Lorsque le milieu n'est pas parfaitement élastique (cas du soussol), l'onde sismique subit en plus une dissipation d'énergie couramment nommé amortissement matériel qui est caractérisé par une décroissance exponentielle de l'amplitude de l'onde en exp (- $\alpha r), \alpha$ correspondant au coefficient d'atténuation, couramment utilisé en acoustique. En sismique, il est plus commun d'employer le coefficient de qualité $\mathrm{Q}$. Ce dernier peut être relié au coefficient d'atténuation par la relation suivante (Piwakowski, 1989) :

$$
\alpha=\pi /(\mathrm{Q} \lambda)
$$

$\lambda$ représente la longueur d'onde. Cette dernière dépend de la fréquence de la source $F_{\mathrm{r}}$ et de la vitesse de propagation des ondes de compression $V_{p}$ dans le matériau $\left(\lambda=V_{\mathrm{p}} / \mathrm{F}_{\mathrm{n}}\right)$.

Dans le cas où aucun amortissement n'existe (milieu homogène et élastique), le coefficient $Q$ est infini.

\section{B. Coefficient de détection $C_{\text {dec }}$}

Afin d'analyser les résultats d'une analyse tempsfréquence, la possibilité de détecter une cavité est quantifiée au moyen de trois critères définis par Léonard (2000) :

1) Un critère d'efficacité de la mêthode $E_{c}$

$$
E_{c}=\frac{W_{r}}{W-W_{s}} \text { avec } 0 \leq E_{c} \leq 1
$$

où $W, W_{r}$ et $W_{s}$ (Fig. A1) sont respectivement le nombre total de fenêtres de l'analyse temps-fréquence, le nombre total de fenêtres où la fréquence de résonance de la cavité est observable dans l'analyse temps-fréquence et le nombre total de fenêtres contenant en majorité une information provenant de l'onde de surface. 
Le critère $E_{\text {est maximal lorsque la fréquence de }}$ es résonance de la cavité $\mathrm{f}_{\mathrm{d}}$ est observable dans toutes les fenêtres où l'onde de surface ne domine pas. Plus le critère $\mathrm{E}_{\mathrm{c}}$ augmente, plus la cavité est facilement détectable.

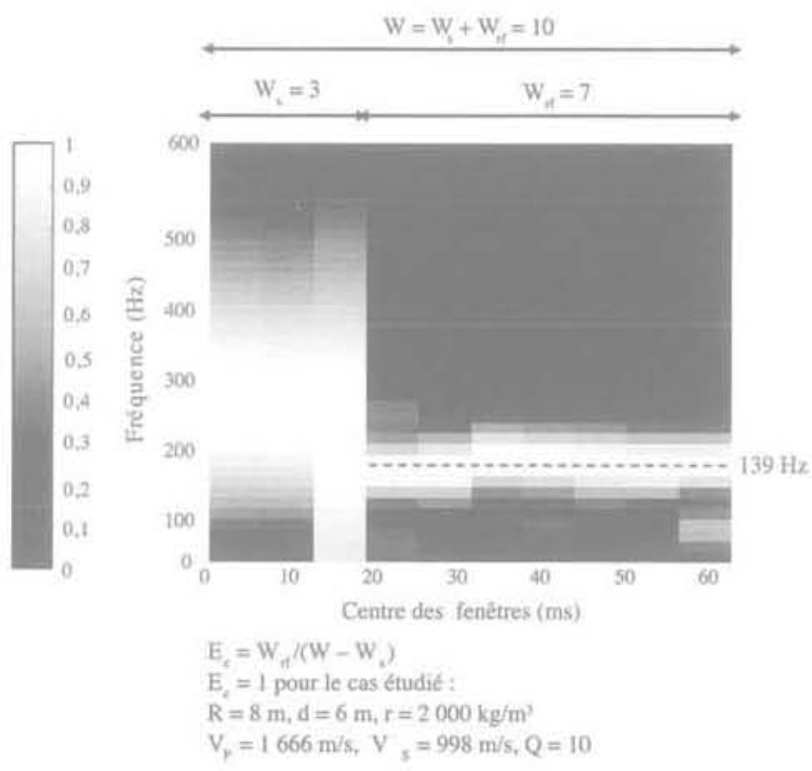

FIG. B. Illustration des paramètres introduits dans le critère de détection $\mathrm{C}_{\text {dec }}$ : le critère $\mathrm{E}_{c}$. La cavité est modélisée par un vide cylindrique de rayon $\mathrm{R}=8 \mathrm{~m}$ localisé dans le sol à une profondeur $\mathrm{d}=6 \mathrm{~m}\left(\mathrm{p}=2000 \mathrm{~kg} / \mathrm{m}^{3}, \mathrm{~V}_{\mathrm{p}}=\right.$ $1666 \mathrm{~m} / \mathrm{s}, V_{\mathrm{s}}=998 \mathrm{~m} / \mathrm{s}, \mathrm{Q}=10$ ).

2) La bande passante à - $3 \mathrm{db} \Delta$ f (Fig. B.2)

Ce critère correspond à la distance entre les points d'un même pic ayant pour amplitude 0,7 fois la valeur maximale de ce pic. Ce critère est évalué pour le pic obtenu à la fréquence de résonance de la cavité $f_{f}$ dans la fenêtre $Y_{K}(f)$ sélectionnée.

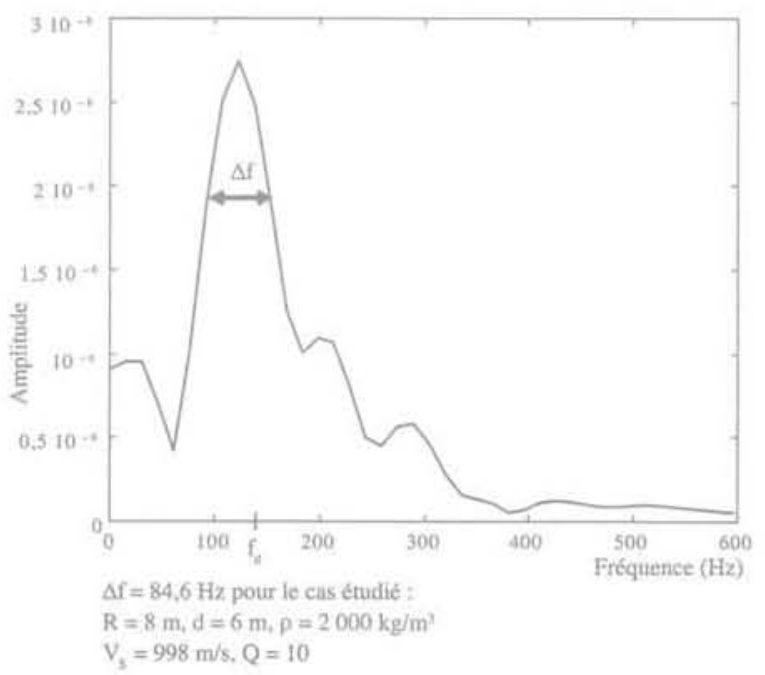

FIG. B. . Illustration des paramètres introduits dans le critère de détection $\mathrm{C}_{\text {dec }}$ : la bande passante à - $3 \mathrm{~dB} \Delta \mathrm{f}$.

La cavité est modélisée par un vide cylindrique de rayon $\mathrm{R}=8 \mathrm{~m}$ localisé dans le sol à une profondeur $\mathrm{d}=6 \mathrm{~m}\left(\mathrm{p}=2000 \mathrm{~kg} / \mathrm{m}^{3}, \mathrm{~V}_{\mathrm{p}}=\right.$ $1666 \mathrm{~m} / \mathrm{s}, V_{\mathrm{s}}=998 \mathrm{~m} / \mathrm{s}, \mathrm{Q}=10$ ).
3) Le nombre d'harmonique $\mathrm{NH}$ de la fréquence de résonance de la cavité $\mathrm{f}_{\mathrm{d}}$ (Fig. B.3)

Le paramètre NH comptabilise le nombre de pics apparaissant dans la fenêtre $Y_{k}$ (f) sélectionnée provenant des harmoniques de $f_{\text {df. }}$ Dans le cas présenté à la figure B.3, le paramètre $\mathrm{NH}$ est égal à 3 , puisque 3 pics sur 4 observables sont à une fréquence $f_{d}$ modulo $f_{t}$

Afin de résumer les variations de ces trois critères, nous introduisons le critère de détection d'une cavité sous la forme:

$$
\mathrm{CDEC}=(\mathrm{NH} . \mathrm{EC}) / \Delta f
$$

$\mathrm{NH}$ et $\Delta$ f ont été normalisés par rapport à leurs valeurs maximales respectives obtenues dans l'ensemble des configurations présentées dans (Léonard, 2000), soient respectivement 6 et $615 \mathrm{~Hz}$.

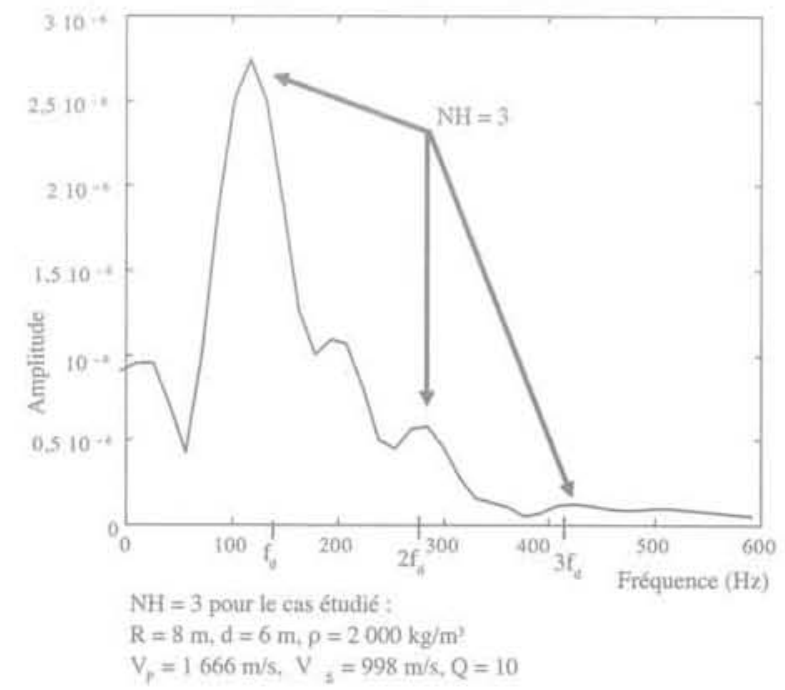

FG. 8.3 Illustration des paramètres introduits dans le critère de détection $\mathrm{C}_{\mathrm{dec}}$ : le critère $\mathrm{NH}$. La cavité est modélisée par un vide cylindrique de rayon $R=8 \mathrm{~m}$ localisé dans le sol à une profondeur $d=6 \mathrm{~m}\left(\rho=2000 \mathrm{~kg} / \mathrm{m}^{3}, V_{p}=\right.$ $1666 \mathrm{~m} / \mathrm{s}, V_{\mathrm{s}}=998 \mathrm{~m} / \mathrm{s}, \mathrm{Q}=10$ ).

\section{Coefficient d'anomalie AN}

Afin d'exprimer la dimension de la cavité en terme d'imagerie acoustique, Piwakowski et al. (1998) ont introduit un coefficient d'anomalie AN. Pour faciliter la lecture de cet article, nous rappelons sa définition.

II est communément admis que chaque géophone "cvoit $»$ à une profondeur donnée d une certaine surface circulaire $S_{\text {, de rayon }} R_{f}=[\mathrm{d} \lambda / 2]^{0.5}$, définit comme étant la surface de Fresnel ( $\lambda$ étant la longueur d'onde du signal sismique enregistré). Le rayon $\mathrm{R}$, de cette surface est communément interprété comme la résolution latérale de l'image sismique (Mari et al., 1998).

Ainsi, le dispositif sismique obtenu par traitement ne voyant que la zone de Fresnel, l'anomalie causée par la présence d'une cavité sera proportionnelle au rapport $\mathrm{S} / \mathrm{S}, \mathrm{S}$ étant la surface de la cavité contenue dans Sf (Fig. C.1). Le coefficient de détection AN est alors défini comme le rapport suivant:

$$
\begin{gathered}
\mathrm{AN}=\mathrm{S}_{\mathrm{c}} / \mathrm{S}_{\mathrm{f}} \text { pour } \mathrm{S}_{\mathrm{c}}<\mathrm{S}_{f} \\
\mathrm{AN}=1 \text { pour } \mathrm{S}_{\mathrm{c}}>\mathrm{S}_{\mathrm{f}}
\end{gathered}
$$

Plus la taille de l'objet augmente, plus le rapport $\mathrm{AN}$ est grand. D'après ce modèle, si $\mathrm{S}_{\mathrm{c}}$ devient égale à $\mathrm{S}_{p}$ 
alors $\mathrm{AN}=1$ ce qui signifie que la cavité se comporte comme une couche infinie. Pour $\mathrm{S}_{c}<\mathrm{S}_{p}$, le coefficient d'anomalie AN fournit une dimension de la cavité qui est fonction de la structure géologique (dont la longueur d'onde $\lambda$ dépend).

De nombreuses études numériques ont confirmé l'utilité d'un tel coefficient: Piwakowski et al. (1998), Léonard (2000), Piwakowski et al. (2002), Driad et Piwakowski (2002). Dans la plupart des cas, une cavité caractérisée par un coefficient AN proche de 0,5 peut être détectable par sismique réflexion. Ce critère ( $A N \geq 0,5)$ ne peut pas être considéré comme un seuil absolu. Il peut changer en fonction des conditions géologiques du site.

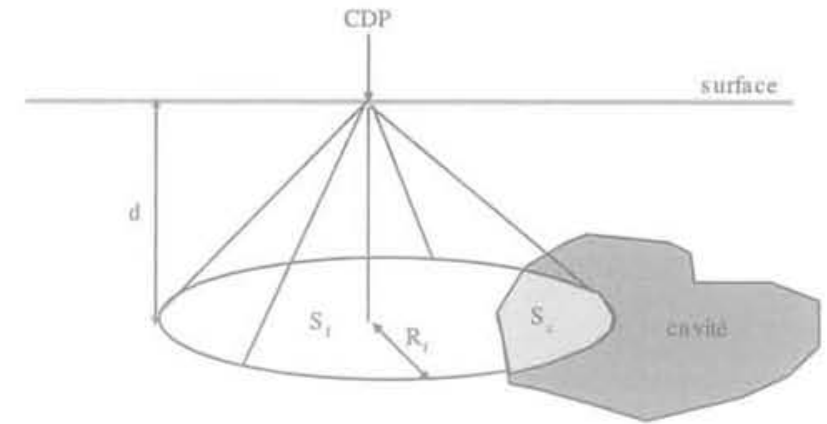

FG, CI Concept du coefficient d'anomalie AN.

\section{Bibliographie}

Abraham O., Léonard C., Còte P., Piwakowski B. - Time-frequency analysis of impact-echo signals: Numerical modeling and experimental validation. $\mathrm{ACl}$ Material Journal, novembre-décembre 2000, p. 645-657.

Abraham O.. Cóte P. - Thickness frequency profile for the detection of voids in tendon duct. ACI Structural Journal, 99 (3), 2002, p. 239-247.

Abraham O.. Leparoux D. - Détection de cavités non maçonnées à l'aide d'ondes de surface. Projet national Criterre Thème 1, Rapport n², 2003.

Akl K., Richards P.G. - Quantitative Seismology, Theory and Methods. W.H. Freeman and Co., San Francisco, 1980.

Beres M., Luetscher M., Olivier R. - Integration of ground penetrating radar and microgravimetric methods to map shallow caves. Journal of Applied Geophysics, 46, 2001, p. 249-262.

Bracewell R. - The Fourier transform and its applications. McGraw-Hill, NY 1965.

Branham K.L., steeples D.W. - Cavity detection using high resolution seismic methods. Mining Engineering, 40, 1988, p. 115-119.

Branston M.W. Styles P. - The application of time-lapse microgravity for the investigation and monitoring of subsidence at Norwich. Cheshire. The Quarterly Journal of Engineering Geology and Hydrogeology, 36, 3, 2003, p. 231-244.

Carino N.J., Sansalone M., Hsu N. - Flaw detection in concrete by frequency spectrum analysis of impact-echo waveforms. Int. Advances in Non-destructive Testing, 12, 1986, p. 117-146.

Chamberlain A.T., Andrew T.C., William S. Chis P. Roslyn C. - Cave detection in limestone using ground penetrating radar. Journal of Archaeological Science, 27, 2000, p. 957-964.

Cook J.C. - Seismic mapping of underground cavities using reflection amplitude. Geophysics, 30, 1965, p. 527-538.
Crawford, N.C. - Microgravity investigations of sinkhole collapses under highways. First international conference on the application of geophysical methodologies to Transportation facilities and infrastructures, St Louis; Missouri, décembre 2000.

Driad L., Piwakowski B. - Detection and characterisation of underground cavities using high resolution seismic (HRSR), $8^{\text {th }}$ Congress of European Section Environmental \& Engineering Geophysical Society, Lisboa, 2002.

Fauchard C.. Pothérat P. - Détection de cavités souterraines par méthodes géophysiques. Guide technique, collection "Techiniques et méthodes des laboratoires des ponts et chausséesm, LCPC. 2004. $170 \mathrm{p}$.

Forde M.C., Clark M., Halliday J., Watson J. - Guidance on the use of NDE on voided P-T concrete bridge beams using impact echo. Proc TRB $82^{\text {nd }}$ Meeting, Washington, DC, USA, January 2003.

Gutkowski T.G., Dym C.L. - Propagation of ground vibration; a review. Journal of Sound and vibration, 49, 1976, p. 173179 .

Kahan M. - Non-destructive structure evaluation: an application of seismic signal analysis. MSc in Mechanical Engineering, MIT, USA, 1993

Kharrat Y. - Auscultation des structures en béton par tomographie sonique de vitesse et d'atténuation. Thèse, Université de Sherbrooke, Canada, 1997.

Lavergne M. - Méthodes sismiques. Publication de l'Institut français du pétrole, Edition Technip, Paris, 1986.

Leparoux D., Bitri A, Grandjean G. Underground cavities detection: a new method based on seismic Rayleigh waves. European Journal of Environmental \& Engineering Geophysical, 5, 2000, p. 33-53.

Lin .I.M. Sansalone M. - Impact-echo response of hollow cylindrical concrete structures surrounded by soil and rock Part I: Numerical studies. Geotechnical Testing Journal, 17 (2), 1994a, p. 207-219.

Lin J.M., Sansalone M. - Impact-echo response of hollow cylindrical concrete structures surrounded by soil and rock. Part II : Experimental studies. Geotechnical Testing Journal, 17 (2), 1994b, p. 220-226.

Léonard C. - Détection des cavités souterraines par rêflexion sismique haute résolution et par impac écho. Thèse, Université des sciences et technologies de Lille. 2000

Mari J.L, Glangeaud F., Coppens F. - Traitement du signal pour géologues et géophysiciens. Publication de I'Tnstitut français du pétrole, Edition Technip, Paris, 1997.

Mari J.L., Arens G., Gaudiani P., Chapellier D. - Méthodes de géophysique de gisement appliquées au génie civil. Publication de l'Institut français du pétrole. Édi. tion Technip, Paris, 1998.

Miller R.D., Pullan S.E., Waldner J.S. Haeni F.P. - Field comparison of seismic sources. Geophysics, 51, 1986, p. 2067. 2092

Moreaux D. - Localisation de cavités sou. terraines par réflexion sismique haute résolution : êtude numérique, étude de terrain. Thèse, Université des sciences et technologies de Lille, France. 1997.

Ormos T. Gyulai A., Nuari Z. - Cavity detection with resistivity and shallow seismic methods. $V^{\text {th }}$ Meeting of the Environmental and Engineering Geophysical Society, Budapest. Hungary, 1999.

Piwakowski. B. - Contribution à la théorie de la réponse impulsionnelle de diffraction. Exemple d'application : détection sismique de cavités souterraines. Habilitation à diriger des recherches, université de Valenciennes, 1989.

Piwakowski B., Shahrour I. - Imagerie du proche sous-sol par sismique haute 
résolution : application à la détection des cavités, Bull. de liaison des lab. des ponts et chaussés, 214, 1998, p. 19-29.

Piwakowski B., Léonard C., Shahrour I. La sismique réflexion haute résolution. un outil pour la reconnaissance des couches superficielles, Revue française de géotechnique, 101, 2002, p. 23-33.

Rybakov M., Goldshmidt V., Fleischer L. Rotstein, Y, - Cave detection and 4-D monitoring: a microgravity case history near the Dead Sea. The Leading Edge, $20,8,2001$, p. $896-900$.
Ricker N.H. - Transient waves in visco-electric media. Elsevier Scientific Publishing Company, 1977.

Shahrour I. - PECPLAS: A finite element software for the resolution of geotechnical problems. International Symposium on Geotechnical/Information Sciences, Presses des. Ponts et Chaussées, Paris, 1992, p. 327-334.

Sansalone M., Carino N.J. - Impact-echo method, Concrete International, 1988, p. 38-46.

Sansalone M., Streett W.B. - Impact-echo: non-destructive testing of concrete and masonry. Bulbrier Press, Ithaca, N.Y, 1997.

Van Schoor M. - Detection of sinkholes using 2D electrical resistivity imaging. Journal of Applied geophysics, 50, 2002, p. 393-399.

Williams T.J., Sansalone M. Streett W.B. Poston R.W., Whitlock A.R. - Non-destructive evaluation of masonry structures using the impact-echo method. The Masonry Journal, 15(1), 1997, p. 47-57.

Yilmaz O. - Seismic Signal Processing. Society of Exploration Geophysicists, 1987. 